\title{
The Extracellular Matrix of Plants: \\ Molecular, Cellular and Developmental Biology
}

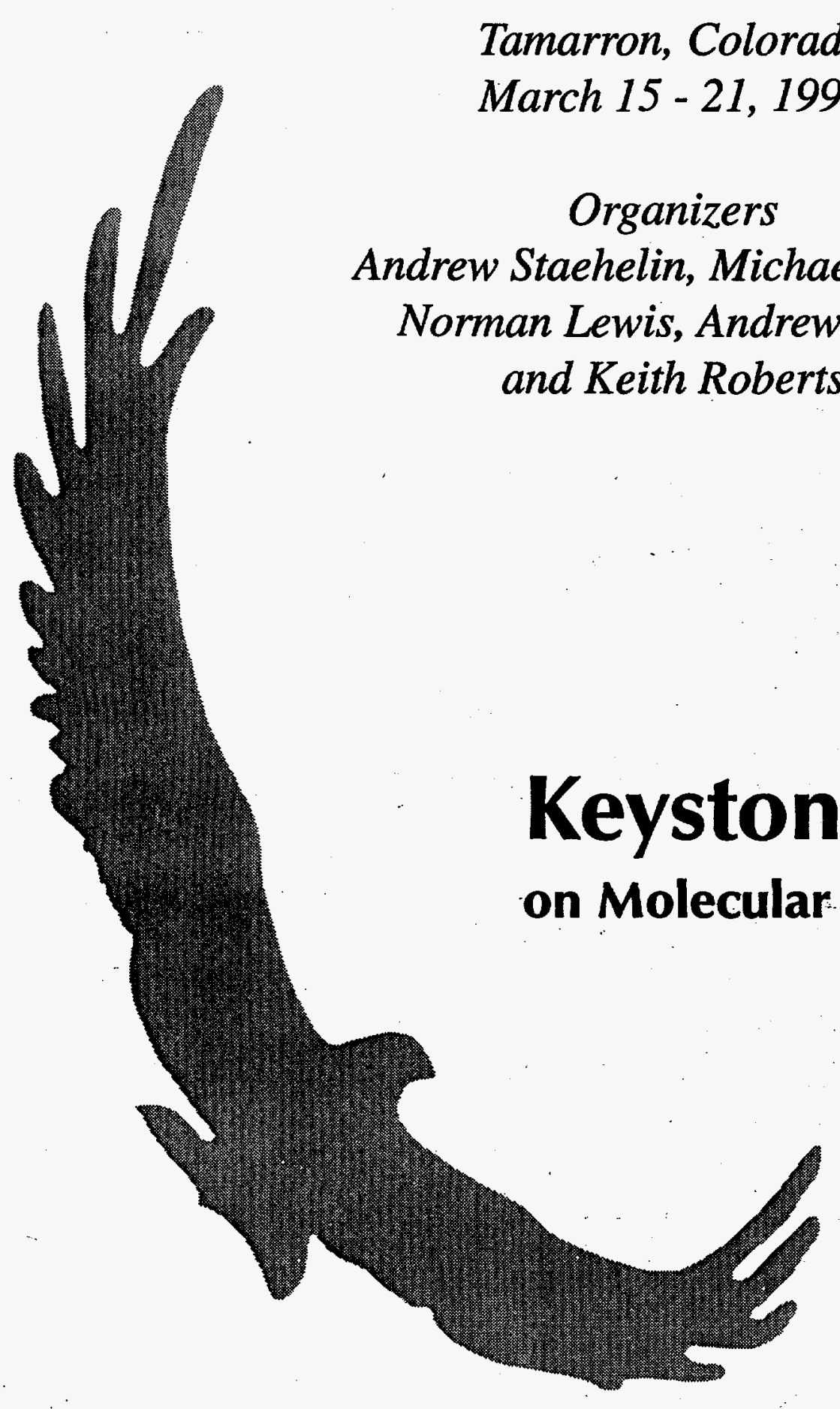

MASTER

\author{
Tamarron, Color
}

Organizers

Norman Lewis, Andrew Mort

and Keith Roberts

\section{Keystone Symposia on Molecular and Cellular Biology}

Drawer 1630 - Silverthorne, CO 80498

(970) 262-1230 - Fax: (970) 262-1525

e-mail: keystone@symposia.com - web site: http://www.colorado.net/symposia 


\section{DISCLAIMER}

This report was prepared as an account of work sponsored by an agency of the United States Government. Neither the United States Government nor any agency thereof, nor any of their employees, make any warranty, express or implied, or assumes any legal liability or responsibility for the accuracy, completeness, or usefulness of any information, apparatus, product, or process disclosed, or represents that its use would not infringe privately owned rights. Reference herein to any specific commercial product, process, or service by trade name, trademark, manufacturer, or otherwise does not necessarily constitute or imply its endorsement, recommendation, or favoring by the United States Government or any agency thereof. The views and opinions of authors expressed herein do not necessarily state or reflect those of the United States Government or any agency thereof. 


\section{DISCLAIMER}

Portions of this document may be illegible in electronic image products. Images are produced from the best available original document. 
A Keystone Symposium

THE EXTRACELLULAR MATRIX OF PLANTS:

MOLECULAR, CELLULAR AND DEVELOPMENTAL BIOLOGY

Organizers: Andrew Staehelin, Michael Hahn, Norman Lewis, Andrew Mort and Keith Roberts

March 15-21, 1996; Tamarron, Colorado

\section{Friday, March 15}

$\begin{array}{ll}2-7 \mathrm{pm} & \text { Registration } \\ \text { 8-8:30pm } & \text { Orientation } \\ \text { 8:30-9:30pm } & \text { Keynote Address } \\ & \text { Andrew Staehelin * University of Colorado } \\ & \text { John Lowe University of Michigan } \\ & \text { "Design and Properties of Glycosyltransferases" }\end{array}$

Alcove

Silverton Center

\section{Saturday, March 16}

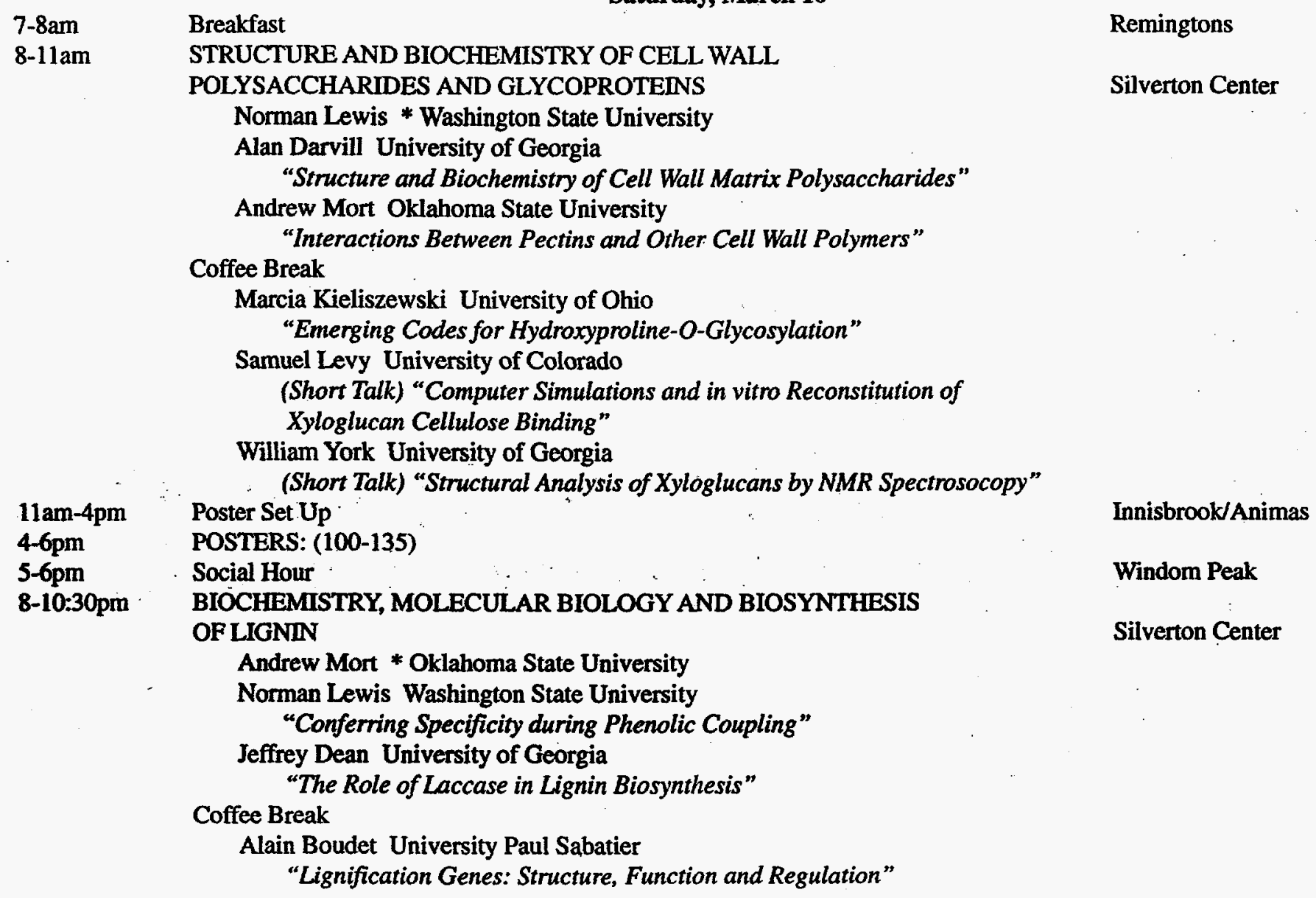

Innisbrook/Animas

Windom Peak

Silverton Center

\section{Sunday, March 17}

$\begin{array}{lll}\text { 7-8am } & \text { Breakfast } & \text { Remingtons } \\ \text { 8-1lam } & \text { BIOSYNTHESIS I: SECRETORY PATHWAY AND SYNTHESIS OF } & \text { Silverton Center } \\ \text { GLYCOPROTEINS } & \\ \text { Deborah Delmer * University California-Davis } & \\ \text { Andrew Staehelin University of Colorado } & \text { "The Plant Golgi Apparatus and Cell Plate Formation" } \\ \text { Sabine Waffenschmidt University of Koln } & \\ \text { "Biosynthesis of Structural Cell Wall Glycoproteins" } & \\ \text { Coffee Break } & \text { Nicholas Carpita Purdue University } \\ \text { "Biosynthesis of Matrix Polysaccharides" }\end{array}$


4-6pm

5-6pm

8-10:30pm
Ariel Orellana University of Chile

\section{Sunday, March 17 (cont.)}

(Short Talk) "Transport of Nucleotide Sugars for Xyloglucan Synthesis into Golgi Vesicles"

Debra Mohnen University of Georgia

(Short Talk) "Biosynthesis of Pectin: Solubilization of Polygalacturonate 4- $\alpha$ -

Galacturonosyl Transferase Activity from Tobacco Membranes"

POSTERS: (100-135)

Social Hour $\quad$ Windom Peak

BIOSYNTHESIS II: MATRIX POLYSACCHARIDES, CALLOSE, AND CELLULOSE Silverton Peak

Nicolas Carpita * Purdue University

Deborah Delmer Hebrew University

"Probing the Subunit of the $\beta$-Glucan Synthase Complexes of Cotton Fibers"

Bruce Wasserman Rutgers University

"Molecular Architecture of Plasma Membrane Callose Biosynthetic Complexes"

Coffee Break

Katia Ruel CNRS

(Short Talk) "Lignin Biogenesis in Higher Plant Cell Walls - An Investigation by

Non-invasive Techniques"

John Ralph US Dairy Forage Research Center

(Short Talk) "Cell Wall Cross-linking in Grasses by Ferulates and Dehyrodiferulates" 7-8am

8-11am

$11 \mathrm{am}-4 \mathrm{pm}$

$1-4 \mathrm{pm}$

4-6pm

$5-6 \mathrm{pm}$

8-10:30pm

\section{Monday, March 18}

Breakfast

ROLE OF THE EXTRACELLULAR MATRIX IN PLANT GROWTH

AND DEVELOPMENT I

Ralph Quatrano * University of North Carolina

Keith Roberts John Innes Centre

"Assembly of the Extracellular Matrix of Plants"

Adrienne Clarke University of Melbourne

"Characterization of Four Glycoconjugates From Pistils of Nicotiana

Coffee Break alata Containing Arabinose and Galactose Substituents"

Daniel Cosgrove Pennsylvania State University

"Characterization of Expansins and Their Role in Plant Cell Enlargement"

Richard Blanton Texas Technology University (Short Talk) "Patterns of Cellulose Depositions by Dictyostelium Discovered during Normal and Abnormal Development"

Sarah Whitney Unilever Research (Short Talk) "In vitro Construction of Cellulose/Hemicellulose Networks: Ultrastructural and Molecular Aspects"

Poster Set Up

Innisbrook/Animas

Workshop: GLYCOCHAPERONES

(Workshop sponsored by: The National Science Foundation)

Lawrence Griffing * Texas A\&M University

"Glycochaperons - A Useful Interkingdom Paradigm?"

Daniel Hebert Yale University

"The Roles of Calnexin and Calreticulin, Lectin Chaperones of the ER"

Peter Scheiffele EMBL

"N-glycosylation as a Sorting Signal for Intracellular Transport"

Clifton Barry III NIAID

"The Role of Carbohydrate in Mycobacterial Cell Wall Structure and Assembly"

Mini Talks and General Discussion

POSTERS: (200-237)

Social Hour

ROLE OF EXTRACELLULAR MATRIX IN PLANT CELL GROWTH

AND DEVELOPMENT II

Daniel Cosgrove * Pennsylvania State University

Wolf-Dieter Reiter University of Connecticut

"Genetic Analysis of Cell Wall Synthesis in Arabidopsis"
Remingtons

Silverton Center 
Monday, March 18 (cont.)

Alan Bennett University California-Davis

"Role of Endo-1, 4-Glucanase Gene Family Members in Cell Growth and Expansion"

Coffee Break

Alice Cheung Yale University

(Short Talk) "A Transmitting-specific Extracellular Glycoprotein Promotes

and Attracts Pollen Tube Growth"

Mary Tierney University of Vermont

(Short Talk) "Molecular Dissection of the Wound Response for Two Soybean Extracellular Matrix Proteins"

Tuesday, March 19

7-8am

8-11am

4-6pm

$5-6 \mathrm{pm}$

8-10:30pm

Breakfast

AND DEVELOPMENT III

Keith Roberts * John Innes Centre

Ralph Quatrano University of North Carolina

Colin Brownlee Marine Biological Association

Coffee Break

Michael Hahn University of Georgia

"Oligoglucoside Elicitor Perception in Soybean"

"Oligochitin Elicitor Perception in Rice"

POSTERS: (200-237)

Social Hour
ROLE OF EXTRACELLULAR MATRIX IN PLANT CELL GROWTH

"Cytoskeletal-Cell Wall Interactions in the Fixation of Polarity"

"Cell Wall Control of Cell Fate in Fucus"

PLANT CELL WALLS IN SYMBIOSIS AND PATHOGENESIS I

Naoto Shibuya National Institute of Agricultural Research

PLANT CELL WALLS IN SYMBIOSIS AND PATHOGENESIS II

Herman Spaink Leiden University

"Signal Molecules Which Induce Plant Organogenesis"

Edward Farmer University of Lausanne

"Biological Regulators from the Pectic Matrix and their Interaction with the Cell Surface"

Coffee Break

Bruno Moerschbacher University of Munster

(Short Talk) "Surface Signals in Rust-infected Wheat"

Paul Bolwell University of London

(Short Talk) "Modelling Cell Wall Changes during Defense"
Windom Peak

Silverton Center
Remingtons

Silverton Center

\section{Wednesday, March 20}

7-8am

8-10:30am

Breakfast

PLANT CELL WALLS IN SYMBIOSIS AND PATHOGENESIS III

Ronald Sederoff * North Carolina State University

Giulia De Lorenzo University of Rome

"Polygalacturonase-Inhibiting Protein (PGIPs) Plant Cell Wall Proteins

Specialized for Pathogen Recognition"

Christopher Lamb Salk Institute

"Mechanism and Function of the Oxidative Burst in Plant Disease Resistance"

Coffee Break

Bernard Fritig CNRS

"Pathogenesis-Related Proteins: Biological Activities and Roles in Plant-Microbe Interactions"

10:30-11am

CELL WALLS IN FOOD, FEED AND INDUSTRY I

Grant Reid University of Stirling

"Characterization of Enzymes Associated with Xyloglucan Metabolism"

2-4:30pm CELL WALLS IN FOOD, FEED AND INDUSTRY II

Session Sponsored by: Hercules Incorporated

Alan Darvill * University of Georgia

Alphons Voragen Wageningen Agricultural University

"Use of Cell Wall Degrading Enzymes in Food Processing"
Remingtons

Silverton Center 


\section{$5: 30-6: 30 \mathrm{pm}$ \\ $6: 30-9: 30 \mathrm{pm}$ \\ $8-9 \mathrm{pm}$}

$9 \mathrm{pm}-12 \mathrm{am}$
Wednesday, March 20 (cont.)

Dean Della Penna University of Arizona

"A New Class of Aromatic Cell Wall Proteins Involved in Pectin Metabolism"

Coffee Break

John Bedbrook DNA Plant Technology Corporation

"Control of Cell Wall Disassembly in Ripening Fruit and

Commercialization of Research Results"

Social Hour

Banquet Silverton Center

Banquet Address

Bruce Stone ${ }^{*}$ La Trobe University

Peter Albersheim University of Georgia

"Cell Wall: Where We Are and Where We Are Going"

Entertainment

Departure

Thursday, March 21

We gratefully acknowledge support from:

Agracetus, Inc.

Campbell Soup Company

CIBA-GEIGY Corporation

Hercules Incorporated

The Samuel Roberts Nobel Foundation, Inc.

Northrup King Company

Pioneer Hi-Bred International, Inc.

Sandoz Agro, Inc.

Sandoz Seeds, Ltd.

Science \& Technology Agency, Japan

This meeting was also supported in part by:

U.S. Department of Energy

Grant No. DE-FG03-95ER20210

Glycochaperone Workshop supported, in part, by a grant from the

National Science Foundation, MCB 9630128 
The Extracelluar Matrix of Plants: Molecular, Cellular and Developmental Biology (D5)

\section{Speaker List}

\begin{abstract}
PETER ALBERSHEIM COMPLEX CARBOHYDRATE RSCH UNIV OF GEORGIA 220 RIVERBEND RD ATHENS GA 306024712 706-542-4404
\end{abstract}

JOHN BEDBROOK PLANT GENETICS DNA PLANT TECHNOLOGY CORP 6701 SAN PABLO AVE OAKLAND CA 94608 510-547-2395

ALAN BENNETT
VEGETABLE CROPS
UNIV CALIFORNIA-DAVIS
MANN LABORATORY
DAVIS CA 95616
916-752-6730
RICHARD BLANTON
BIOLOGICAL SCIENCES
TEXAS TECH UNIVERSITY
PO BOX 43131
LUBBOCK TX 794093131
806-742-2704

G. PAUL BOLWELL BIOCHEMISTRY UNIV OFLONDON ROYAL HOLLOWAY EGHAM HILL SURREY TW20 OEX UK

178-444-3553

\section{ALAIN BOUDET \\ PLANT PHYSIOL/LA NO 241 \\ UNIV PAUL SABATIER \\ 118 RUE DE NARBONNE \\ TOULOUSE CEDEX 31062 \\ FRANCE \\ 336-155-6754}

COLIN BROWNLEE

MARINE BIOLOGICAL ASSOC LAB CITADEL HILL PLYMOUTH PL1 2PB UK

175-222-2772

$$
\text { (as of } 1 / 12 / 96 \text { ) }
$$

NICHOLAS CARPITA BOTANY \& PLANT PATH

PURDUE UNIVERSITY LILLY HALL OF LIFE SCIENCE WEST LAFAYETTE IN 47907 317-494-4653

ALICE CHEUNG

YALE UNIVERSITY

PO BOX 208104

NEW HAVEN CT 065208104

203-432-3890

ADRIENNE E. CLARKE

BOTANY

UNIV OF MELBOURNE

PARKVILLE VICTORIA 3052

AUSTRALIA

613-344-5043

DANIEL COSGROVE

BIOLOGY

PENNSYLVANIA STATE UNIVER-

STTY

208 MUELLER LAB

UNIVERSITY PARK PA 16802

814-863-3892

ALANDARVILL COMPLEX CARBOHYDRATE RSCH UNIV OF GEORGIA 220 RIVERBEND ROAD ATHENS GA 306024712

706-542-4411

\section{GIULIA DE LORENZO}

BIOLOGIA VEGETALE

UNIV DE ROMA-LA SAPIENZA PIAZZALE ALDO MORRO 5

ROME 00185

ITALY

396-445-2237

JEFFREY DEAN

BIOCHEM \& MOLEC BIOLOGY UNIV OF GEORGIA ATHENS GA 306027229

706-542-1710
DEAN DELLA PENNA PLANT SCIENCES UNIV OF ARIZONA 303 FORBES HALL TUCSON AZ 85721 520-621-8725

DEBORAH DELMER BOTANY HEBREW UNIVERSITY INST OF LIFE SCIENCES GIVARIT RAM/JERUSALEM 91904 ISRAEL

2-658-5235

EDWARD FARMER

IBPV

UNIV OF LAUSANNE

BIOLOGY BLDG

LAUSANNE 1015

SWITZERLAND

21-692-4190

BERNARD FRITIG

IBMP

CNRS

12 RUE DU GENERAL ZIMMER

STRASBOURG CEDEX 67000

FRANCE

338-841-7280

MICHAEL HAHN

COMPLEX CARBOHYDRATE RSCH

UNIV OF GEORGIA

220 RIVERBEND RD

ATHENS GA 306024712

706-542-4457

MARCIA KIELISZEWSKI

CHEMISTRY

OHIO UNIVERSITY

271-C CLIPPINGER HALL

ATHENS OH 45701

614-593-9466

CHRISTOPHER LAMB

PLANT BIOLOGY LAB

SALK INSTITUTE

PO BOX 85800

SAN DIEGO CA 921865800

619-453-4100 


\section{Speaker List (cont.)}

SAMUEL LEVY

MCD BIOLOGY

UNIV OF COLORADO

BOULDER CO 803090347

303-492-8893

NORMAN LEWIS

INST BIOLOGICAL CHEMISTRY

WASHINGTON STATE UNIVERSITY

467 CLARK HALL/PO B 6340

PULLMAN WA 99164

509-335-2682

JOHN LOWE

HOWARD HUGHES MED INST

UNIV OF MICHIGAN

1150 W MED CTR DR/MSRBI

RM3510

ANN ARBOR MI 481090650

313-747-4779

BRUNO MOERSCHBACHER

BIOCHEMISTRY OF PLANTS

UNIV OF MUNSTER

HINDENBURGPLATZ 55

MUNCHEN D 48143 GERMANY

24-180-5812

DEBRA MOHNEN

BIOCHEM/COMPLEX CARB RSCH

UNIV OF GEORGIA

220 RIVERBEND ROAD

ATHENS GA 30602

706-542-4458

ANDREW MORT

BIOCHEMISTRY

OKLAHOMA STATE UNIVERSITY

STILLWATER OK 740780454

405-744-6197

ARIEL OREELLANA

BIOLOGY

UNIV OF CHILE

LAS PALMERAS 3425

SANTIAGO CASILLA 653

CHILE

562-678-7247

RALPH QUATRANO

BIOLOGY

UNIV OF NORTH CAROLINA

COKER HALL CB\#3280

CHAPEL HILL NC 275993280

919-962-2098
JOHN RALPH

USDA

US DAIRY FORAGE RESEARCH CTR

1925 LINDEN DR WEST

MADISON WI 53705

608-264-5407

\section{J.S. GRANT REID}

BIOLOGICAL/MOLECULAR SCI

UNIV OF STIRLING

STIRLING SCOTLAND FK9 4LA

UK

178-646-7762

WOLF-DIETER REITER

MOLECULAR CELL BIOLOGY

UNIV OF CONNECTICUT

75 N EAGLEVILLE RD/BOX U-125

STORRS CT 062693125

860-486-5733

KEITH ROBERTS

CELL BIOLOGY

JOHN INNES INSTITUTE

COLNEY LANE

NORWICH NR4 7UH

UK

160-345-2571

KATIA RUEL

CERMAV

CENTRE NATIONAL DE LA RSCH

SCI

BP 53

GRENOBLE CEDEX 0938041

FRANCE

337-654-1145

NAOTO SHIBUYA

CELL BIOLOGY

NATIONAL INST AGROBIOLOGY

RSCH

2-1-2 KANNONDAI TSUKUBA

IBARAKI 305

JAPAN

29-838-8364

HERMAN SPAINK

WASSENAARSEWEG 64

LEIDEN 2333 AL

NETHERLANDS

317-127-5055
ANDREW STAEHELIN

MCD BIOLOGY

UNIV OF COLORADO

CAMPUS BOX 0347

BOULDER CO 803090347

303-492-8843

MARY TIERNEY

BOTANY

UNIV OF VERMONT

120 MARSH LIFE SCIENCE BLDG

BURLINGTON VT 05405

802-656-0434

ALPHONS VORAGEN

FOOD SCIENCE

AGRICULTURAL UNIVERSITY

BOMENWEG 2

WAGENINGEN $6703 \mathrm{HD}$

NETHERLANDS

83-708-3209

SABINE WAFFENSCHMIDT

INST FUR BIOCHEMIE

UNIV OF KOLN

AN DER BOTTMUHLE 2

KOLN 1 D-50678

GERMANY

22-131-1331

BRUCE WASSERMAN

FOOD SCIENCE

RUTGERS UNIVERSITY

COOK COLLEGE

NEW BRUNSWICK NJ 089030231

908-932-9611

SARAH WHITNEY

PLANT TISSUE STRUCTURES

UNILEVER RESEARCH

COLWORTH HOUSE

SHARNBROOK BEDFORD MK42

ORS

UK

123-422-2478

WILLIAM S. YORK

COMPLEX CARBOHYDRATE RSCH

UNIV OF GEORGIA

220 RIVERBEND ROAD

ATHENS GA 306024712

404-542-4487 


\section{Participant List}

(as of 1/12/96)

DEBORAH L. BISHOP

PROGRAM IN MOLECULAR BIOL

UTAH STATE UNIVERSITY

LOGAN UT 843225305

801-797-3497

ROY C BROWN

BIOLOGY

UNIV OF SOUTHWESTERN LOUISI-

ANA

UNIVERSITY AVE/PO 42451

LAFAYETTE LA 705042451

318-482-6757

MICHAEL M. BURRELL

ADVANCED TECHNOLOGIES

SCIENCE PARK/MILTON ROAD

CAMBRIDGE CB4 4WA

UK

122-342-0284

HAZEL CROMBIE

DBMS

UNIV OF STIRLING

STIRLING FK9 4LA

UK

178-647-3171

MARTI DOLATA

CHEMISTRY

OHIO UNIVERSITY

PO BOX 940

ATHENS OH 45701

614-593-9466

MAX DOW

SAINSBURY LABORATORY

JOHN INNES CENTRE

COLNEY LANE

NORWICH NR4 7UH :

UK

160-345-2571

PAUL DUPREE

BIOCHEMISTRY

UNIV OF CAMBRIDGE

TENNIS COURT ROAD

CAMBRIDGE CB2 1QW

UK

122-333-3340
MARY EDWARDS

BIOLOGICAL/MOLEC SCIENCES

UNIV OF STIRLING

STIRLING SCOTLAND FK9 4LA

UK

178-646-7762

JONATHAN GRESSEL

PLANT GENETICS

WEIZMANN INSTITUTE OF SCI-

ENCE

REHOVOT $\amalg-76100$

ISRAEL

8-934-3481

RONALD D. HATFIELD

USDA DAIRY FORAGE RSCH

USDA

1925 LINDEN DR WEST/ARS

MADISON WI 53706

608-264-5407

ARLAND T. HOTCHKISS
ERRC
USDA-ARS
600 E MERMAID LANE
PHILADELPHIA PA 19118
215-233-6448

TADASHI ISHII

BIORESOURCES

FORESTRY \& FOREST PROD RSCH

PO BOX 16 TSUKUBA NORIN

KENKYU DANCHI-NAI IBARAKI

305

JAPAN

029-873-3211

- YUKI ITO

CELL BIOLOGY

NATL INST AGROBIOL RESOURCES

2-1-1 KANNON DAI

TSUKUBA IBARAKI 305

JAPAN

029-838-8364

HANAE KAKU

CELL BIOLOGY

NATIONAL INST AGROBIOLOGY

$\mathrm{RSCH}$

KANNODAI TSUKUBA

IBARAKI 305

JAPAN

29-838-8364
RUTH LAWRENCE

SCH OF BIOLOGICAL SCI

UNIV OF WLAES BANGOR

BANGOR GWYNEDD WALES LL57

2UW

UK

124-835-1151

JUSTIN LEE

INST FUER PFLANZENBIOCHEMIE

WEINBERG 3

. HALLE SAALE D 06120

GERMANY

345-558-2253

BETTY LEMMON

BIOLOGY

UNIV OF SOUTHWESTERN LOUISI-

ANA

UNIVERSITY AVE

LAFAYETTE LA 705042451

318-482-6757

MARIAN LOBLER

INSTITUTE PLANT BIOCHEMISTRY

WEINBERG 3

HALLE D 01620

GERMANY

345-558-2253

MARGARET A. LYNCH

PLANT BIOLOGY

UNIV CALIFORNIA-DAVIS

ROBBINS HALL

DAVIS CA 95616

916-754-8692

JOHN J MACKAY

FORESTRY/GENETICS

NORTH CAROLINA STATE UNIV

PO BOX 8008

RALEIGH NC 27695

919-515-7800

MARIE MANNERLOF

12 CHEMIN DE L HOBIT/BP 27

SAINT SAUVEUR F 31790

FRANCE

336-137-6887 
The Extracelluar Matrix of Plants: Molecular, Cellular and Developme...d Biology (D5)

PETER M. NEUMANN

FACULTY OF AGRICUL ENG

TECHNION

HAIFA 32000

ISRAEL

0-429-3627

LUIS PEREZ-GRAU

CALGENE

1920 FIFTH STREET

DAVIS CA 95616

916-753-6313

IAN POTTER

MOLECULAR/CELL BIOLOGY

UNIV OF CONNECTICUT

BOX U-125

STORRS CT 06269

860-486-2101

ROBERT RABSON

2269 GLENMORE TERRACE

ROCKVILLE MD 20850

301-424-2846

JANE ROBB

MOLEC BIOL \& GENET

UNIV OF GUELPH

GUELPH ONTARIO N1G 2W1

CANADA

519-824-4120

KATIA C RUEL

CERMAV

CENTRE NATIONAL DE LA RSCH SCI BP 53

GRENOBLE CEDEX 0938041

FRANCE

337-654-1145

\section{Participant List (cont.)}

RODNEY A. SAVIDGE

FOREST RESOURCES

UNIV OF NEW BRUNSWICK

BAG SVC \#44555

FREDERICTON NB E3B 6C2

CANADA

506-453-4501

HENRIK V. SCHELLER

PLANT BIOLOGY

ROYAL VETERINARY \& AGRIC

UNIV

40 THORVALDSENSVEJ

FREDERIKSBERG C DK-1871

DENMARK

353-528-3354

ALLAN M. SHOWALTER

PLANT BIOLOGY

OHIO UNIVERSITY

PORTER HALL

ATHENS OH 457012979

614-593-1135

KRISTEN L SMITH

CHEMISTRY

OHIO UNIVERSITY

424 CARRIAGE HILL DRIVE

ATHENS OH 45701

614-593-9466
DERI TOMOS

BIOLOGICAL SCIENCES

UNIV OF WALES

BANGOR GWYNEDD WALES LL57

2UW

UK

124-838-2362

YUZURU TOZAWA

DEPT OF CROP BREEDING

NATIONAL AGRICULTURE RSCH

CTR

3-1-1 KANNONDAI, TSUKUBA

IBARAKI 305

JAPAN

29-838-8949

EDITH P.W.M. VAN DOOREN PLANT BIOTECHNOLOGY TNO CENTER FOR PHYTOTECHNO RULTNO

WASSENAARSEWEG 64

LEIDEN 2333 AL

NETHERLANDS

71-527-4932

ZEV M. WINICUR

MCD BIOLOGY

UNIV OF COLORADO

CAMPUS BOX 347

BOULDER CO 80309

303-492-8893 


\section{D5 REGISTRANTS 1/12/96 OR La IER}

S.JULIET AMBLER

CELL BIOLOGY

JOHN INNES CENTRE

COLNEY LANE/NORWICH

RSCH PARK

NORWICH NORFOLK NR4 7UH

GREAT BRITIAN

60-345-2571

TONY ARIOLI

RSCH SCH FO BIOL PLANT

AUSTRALIAN NATIONAL

UNIVERSITY

GPO BOX 475

CANBERRA A C T 2601

AUSTRALIA

6-249-4446

DAVE ARROWSMTTH

BIOSCIENCES DIVISION

UNILEVER RESEARCH

COLWORTH HOUSE

SHARNBROOK BEDFORD

MK44 1LQ

UK

123-422-2079

\section{GERRIT BELDMAN}

FOOD CHEMISTRY

AGRICULTURAL UNIVERSITY

BOMENWEG 2

WAGENINGEN $6703 \mathrm{HD}$

NETHERLANDS

31-748-3219

\section{DANIELA BELLINCAMPI}

PLANT BIOLOGY

UNIV OF ROME

P ZZA ALDO MORO 5

ROME 00185

TTALY

64-991-2459

MARGOT BERGMANS

FOOD CHEMISTRY

AGRIC. UNIV WAGENINGEN

BOMENWEG 2

WAGENINGEN $6703 \mathrm{HD}$

NETHERLANDS

31-748-4215

IRMA BERNAL-LUGO

BIOQUIMICA

UNIV OF NACIONAL

AUTONOMA

CIUDAD UNIVERSITARIA

MEXICO DF 04510

MEXICO

5-622-5279
DEBORAH L. BISHOP

PROGRAM IN MOLECULAR

BIOL

UTAH STATE UNIVERSITY

LOGAN UT 843225305

801-797-3497

KRISTOPHER A. BLEE

BIOLOGY

UTAH STATE UNIVERSITY

LOGAN UT 843225305

801-797-3497

G. PAUL BOLWELL

BIOCHEMISTRY

UNIV OF LONDON

ROYAL HOLLOWAY

EGHAM HILL SURREY TW20

OEX

UK

178-444-3553

CHRISTOPHER P. BONIN

MOLECULAR/CELL BIOLOGY

UNIV OF CONNECTICUT

75 N EAGLEVILLE ROAD

STORRS CT 06269

860-486-2101

DAVID A. BRMMELL

VEGETABLE CROPS

UNIV CALIFORNIA-DAVIS

MANN LABORATORY

DAVIS CA 95616

916-752-9096

ROY C BROWN

BIOLOGY

UNIV OF SOUTHWESTERN

LOUISIANA

UNIVERSITY AVE/PO 42451

LAFAYETTE LA 705042451

318-482-6757

MICHAEL M. BURRELL ADVANCED TECHNOLOGIES SCIENCE PARK/MILTON ROAD CAMBRIDGE CB4 4WA

UK

122-342-0284

GLADYS I. CASSAB

PLANT MOLECULAR BIOLOGY

NATIONAL UNIV OF MEXICO

BOX 510-3/BIOTECHNOLOGY

INST

CUERNAUACA MORELOS

62271

MEXICO

527-311-4900
FELICE CERVONE

BIOLOGIA VEGETALE

UNIVERSITA DI ROMA LA

SAPIENZA

PIAZZALE ALDO MORO

ROMA 00185

ITALY

64-991-2446

ALEJANDRA A COVARRUBIAS PLANT MOLECULAR BIOLOGY UNIV NACIONAL AUTONOMA MEXICO

APDO POSTAL 510-3

CUERNAVACA MORELOS

62271

MEXICO

$527-311-4700$

CRISTOPHER A. COWAN

MCD BIOLOGY

UNIV OF COLORADO

PORTER BIOSCIENCES

BOULDER CO 803090347

303-492-8893

HAZIEL CROMBIE

DBMS

UNIV OF STIRLING

STIRLING FK9 4LA

UK

178-647-3171

SEAN CUTLER

BIOLOGICAL SCIENCES

STANFORD

290 PARAMA ST

STANFORD CA 94305

415-325-1521

AMY E. DE ROCHER

MSU-DOE/PLANT RSCH LAB

MICHIGAN STATE

UNIVERSITY

EAST LANSING MI 48824

517-353-7876

KATAYOON(KATIE) DEHESH

OILS DIVISION

CALGENE

1920 FIFTH STREET

DAVIS CA 95616

916-753-6313

ALBENA T. DINKOVA-

KOSTOVA

INST OF BIOLOGICAL CHEM

WASHINGTON STATE

UNIVERSITY

PULLMAN WA 991646340

509-335-3435 


\section{D5 REGIs IRANTS 1/12/96 OR L...'ER}

RICHARD A. DIXON

PLANT BIOLOGY DIVISION

SAMUEL ROBERTS NOBLE

FNDN

2510 SAM NOBLE PARKWAY

ARDMORE OK 73402

405-223-5810

MARTI DOLATA

CHEMISTRY

OHIO UNIVERSITY

PO BOX 940

ATHENS OH 45701

614-593-9466

RON LOU DOONG

COMP CARBOHYDRALE RSCH

UNIV OF GEORGIA

220 RIVERBEND ROAD

ATHENS GA 30602

706-542-4499

MAX DOW

SAINSBURY LABORATORY

JOHN INNES CENTRE

COLNEY LANE

NORWICH NR4 7UH

UK

160-345-2571

PAUL DUPREE

BIOCHEMISTRY

UNIV OF CAMBRIDGE

TENNIS COURT ROAD

CAMBRIDGE CB2 1QW

UK

122:333-3340

MARY EDWARDS

BIOLOGICAL/MOLEC

SCIENCES

UNIV OF STIRLING

STIRLING SCOTLAND FK9 4LA

UK

178-646-7762

GEORGES L FREYSSINET

SCIENTIFIQUE

RHONE-POULENC

25. QUAI PAUL DOUIMER

COURBEUQIE CEDEX 92408

FRANCE

14-768-1865

DAVID R. GANG

INST OF BIOLOGICAL CHEM

WASHINGTON STATE

UNIVERSITY

PULLMAN WA 991646340

509-335-2435
BLANCA I. GARCIA-GOMEZ

INST DE BIOTECNOLOGIA

UNAM

APDO POSTAL 510-3

CUERNAVACA MORELOS

62271

MEXICO

$7-311-4700$

IVANA GELINEO

BIOCHEMISTRY/CCRC

UNIV OF GEORGIA

220 RIVERBEND RD

ATHENS GA 30602

706-542-4446

ABIGAIL GREGORY

BIOCHEMISTRY

UNIV OF LONDON

ROYAL HOLLOWAY

EGHAM SURREY ENGLAND

TW20 OEX

UK.

78-444-3559

JONATHAN GRESSEL

PLANT GENETICS

WEIZMANN INSTITUTE OF

SCIENCE

REHOVOT IL-76100

ISRAEI

8-934-3481

MICHAEL R\GRETZ

BIOSCIENCES

MICHIGAN TECHNOLOGICAL UNIV

1400 TOWWNSEND DR ME-EM

BLDG209

HOUGHTON MI 499311295

906-487-3175

MARK J. GRIMSON

BIOLOGICAL SCIENCES

TEXAS TECH UNIVERSITY

BOX 43131

LUBBOCK TX 79409

806-742-2704

CANDACE H. HAIGLER

BIOLOGICAL SCIENCES

TEXAS TECH UNIVERSITY

PO BOX 43131

LUBBOCK TX 794093131

806-742-2707

RONALD D. HATFIELD

USDA DAIRY FORAGE RSCH

USDA

1925 LINDEN DR WEST/ARS

MADISON WI 53706

608-264-5407
PAMELA J. HINES

EDITORIAL

SCIENCE MAGAZINE

1333 H STREET NW

WASHINGTON DC 20005

202-326-6509

ARLAND T. HOTCHKISS

ERRC

USDA-ARS

600 E MERMAID LANE

PHILADELPHIA PA 19118

215-233-6448

TADASHI ISHII

BIORESOURCES

FORESTRY \& FOREST PROD

RSCH

PO BOX 16 TSUKUBA NORIN

KENKYU DANCHI-NAI

IBARAKI 305

JAPAN

029-873-3211

YUKI ITO

CELL BIOLOGY

NATL INST AGROBIOL

RESOURCES

2-1-1 KANNON DAI

TSUKUBA IBARAKI 305

JAPAN

029-838-8364

ALAN M. JONES

BIOLOGY

UNIV OF NORTH CAROLINA

O1OA COKER HALL

CHAPEL HIILL NC 27516

919-962-6932

HANAE KAKU

CELL BIOLOGY

NATIONAL INST

AGROBIOLOGY RSCH

KANNODAI TSUKUBA

IBARAKI 305

JAPAN

29-838-8364

YASUSHI KAWAGOE

BOTANY

HEBREW UNIV OF

JËRUSALEM

JERUSALEM 91904

ISRAEL

2-658-5979 


\section{D5 REGIS RRANTS 1/12/96 OR L_- IER}

KENNETH KEEGSTRA MSU-DOE PLANT RSCH LAB MICHIGAN STATE

UNIVERSITY

EAST LANSING MI 488241312 517-353-2270

THOMAS A. KERR C/O DR BLANTON TEXAS TECH UNIVERSITY BOX 43131

LUBBOCK TX 79409

806-742-2704

PER O. KJELLBOM

PLANT BIOCHEMISTRY

UNIV OF LUND

PO BOX 117

LUND S-220 00

SWEDEN

46-222-4195

BRUCE D. KOHORN

DCMB/LSRC

DUKE UNIVERSITY

BOX 91000

DURHAM NC 277080338

919-613-8183

KLAUS KREUZ

PLANT BIOCHEMISTRY

CIBA-GEIGY LTD

R-1040A.P.21

BASLE CH-4002

SWITZERLAND

61-697-6301

RUTHLAWRENCE

SCH OF BIOLOGICAL SCI

UNIV OF WLAES BANGOR

BANGOR GWYNEDD WALES

LL57 2UW

UK

124-835-1151

ELIZABETH A. LEE

BOTANY

UNIV OF VERMONT

120 MARSH LIFE SCI

BURLINGTON VT 05405

802-656-0894

JUSTIN LEE

INST FUER

PFLANZENBIOCHEMIE

WEINBERG 3

HALLE SAALE D 06120

GERMANY

345-558-2253
BETTY LEMMON

BIOLOGY

UNIV OF SOUTHWESTERN

LOUISIANA

UNIVERSITY AVE

LAFAYETTE LA 705042451

$318-482-6757$

MARIAN LOBLER

INSTITUTE PLANT

BIOCHEMISTRY

WEINBERG 3

HALLE D 01620

GERMANY

345-558-2253

CAROL LOOPSTRA

FOREST SCIENCE

TEXAS A \& M UNIVERSITY

MS 2135

COLLEGE STATION TX

778432135

409-862-2200

ELIZABETHLORD

BOTANY \& PLANT SCI

UNIV CALIFORNIA-RIVERSIDE

RIVERSIDE CA 92521

909-787-4441

VERA LOZOVAYA

CROP SCIENCES

UNIV OF ILLINOIS

1201 W GREGORY

URBANA IL 61801

217-333-9465

MARGARET A. LYNCH

PLANT BIOLOGY

UNIV CALIFORNIA-DAVIS

ROBBINS HALL

DAVIS CA 95616

916-754-8692

JENNIFER W MAC ADAM

PLANTS/SOIL/BIOMETEROL UTAH STATE UNIVERSITY

UMC 4820

LOGAN UT 843324820

801-797-2364

JOHN J MACKAY

FORESTRY/GENETICS

NORTH CAROLINA STATE

UNIV

PO BOX 8008

RALEIGH NC 27695

919-515-7800
SUSAN M. MADRID

DANISCO BIOTECHNOLOGY

LANGEBROGADE 1 PO BOX 17

COPENHAGEN K DK-1001

DENMARK

453-266-2242

MARIE MANNERLOF

12 CHEMIN DE L HOBIT/BP 27

SAINT SAUVEUR F 31790

FRANCE

336-137-6887

ANDREW (MAZZ) MARRY

CELL BIOLOGY

JOHN INNES CENTRE

COLNEY LANE

COLNEY NORWICH NR4 6UH

GREAT BRITIAN

60-345-2571

FIONA M. MC ALISTER

DIV OF PLANT INDUSTRY

CSIRO

GPO BOX 1600

CANBERRA ACT 2617

AUSTRALIA

06-246-5015

MAUREEN MC CANN

CELL BIOLOGY

JOHN INNES INSTITUTE

COLNEY LANE

NORWICH NR4 7UH

UK

446-035-2571

JAMES G. MC CARTHY

FOODS SCIENCES GROUPE

NESTLE RESEARCH \&

DEVELEMENT

201 HOUSATONIC AVE

NEW MILFORD CT 06776

203-355-6447

REGINA S. MC CLINTON

PLANT BIOLOGY

UNIV CALIFORNIA-BERKELEY

111 KOSHLAND HALL

BERKELEY CA 947203102

510-642-0404.

IRVIN J METTLER

BIOTECHNOLOGY

NORTHROP KING CO

317 330TH STREET

STANTON MN 550184300

507-663-7643 


\section{D5 REGIS PRANTS 1/12/96 OR L_. IER}

KNUT MEYER

BIOCHEMISTRY

PURDUE UNIVERSITY

WEST LAFAYETTE IN

479071153

317-494-0651

JORN.DALGAARD MIKKELSEN

DANISCO BIOTECHNOLOGY

1 LAUGEBROGADE

COPENHAGEN DK 1001

DENMARK

453-266-2200

GEOFF M. MTCHELL

BIOCHEMISTRY

ROYAL HOLLOWAY COLLEGE

EGHAM HILL

EGHAM LONDON TW20 0EX

UK

178-444-3549

BRUNO M. MOERSCHBACHER

BIOCHEMISTRY OF PLANTS

UNIV OF MUNSTER

HINDENBURGPLATZ 55

MUNCHEN D 48143

GERMANY

24-180-5812

DEBRA MOHNEN

BIOCHEM/COMPLEX CARB

RSCH

UNIV OF GEORGIA

220 RIVERBEND ROAD

ATHENS GA 30602.

706-542-4458

EGONE MOSINGER

RSCH

SANDOZ AGRO LTD. AGROBIOL RSCH STATION

WITTERSWIL CH4108

SWITZERLAND

161-725-3271

JENIFER M MURPHY

DOE-PLANT RESEARCH LAB

MICHIGAN STATE UNIV

EAST LANSING MI 488241312

517-353-4886

ALLEN K, MURRAY

GLYCOZYME

17935 SKY PARK CIRCLE

SUITE E

IRVINE CA 927146321

714-261-9664
PETER M. NEUMANN

FACULTY OF AGRICUL ENG

TECHNION

HAIFA 32000

ISRAEL

0-429-3627

ANN O'CONNELL

PLANT BIOTECHNOLOGY

ZENECA PLANT SCIENCE

JEALOTTS HILL/RSCH

STATION

BRACKNELL RG12 6EY

UK

134-441-4994

SARA E. PATTERSON

BOTANY

UNIV OF WISCONSIN

430 LINCOLN DRIVE

MADISON WI 53706

608-262-4008

LUIS PEREZ-GRAU

CALGENE

1920 FIFTH STREET

DAVIS CA 95616

916-753-6313

IAN POTTER

MOLECULAR/CELL BIOLOGY

UNIV OF CONNECTICUT

BOX U-125

STORRS CT 06269

860-486-2101

ROBERT RABSON

2269 GLENMORE TERRACE

ROCKVILLE MD 20850

301-424-2846

KEES M. RECOURT

AGROTECHNOLOGICAL RSCH

INST

PO BOX 17

WAGENINGEN 6700 AA

NETHERLANDS

31-747-5227

GLORIA REVILLA

BIOLOGIA

UNIV DE SANTLAGO

LAB FISIOLOGIA VEGETALE

SANTIAGO DE COMPOSTELA

15706

SPAIN

348-156-3100
JANE ROBB

MOLEC BIOL \& GENET

UNIV OF GUELPH

GUELPH ONTARIO N1G 2W1

CANADA

519-824-4120

PETER D. ROBERTS

BIOSORCE TECHNOLOGIES

3333 VACA VALLEY

PARKWAY 1000

VACA VILLE CA 95688

707-446-5501

KATIA C RUEL

CERMAV

CENTRE NATIONAL DE LA

RSCH SCI

BP 53

GRENOBLE CEDEX 0938041

FRANCE

337-654-1145

IMAD N. SAAB

CROP SCIENCES

UNIV OF ILLINOIS

SOUTH II TURNER HALL

URBANA IL 61801

217-333-9743

MARIA SANCHEZ

LAB FISIOLOGIA VEGETAL

UNIV SANTIAGO

SANTIAGO DE

COMPOSTELA/FAC BIO

SANTIAGO DE COMPOSTELA

E 15706

SPAIN

348-156-3100

RODNFY A. SAVIDGE

FOREST RESOURCES

UNIV OF NEW BRUNSWICK

BAG SVC \#44555

FREDERICTON NB E3B 6C2

CANADA

506-453-4501

HENRIK V. SCHELLER

PLANT BIOLOGY

ROYAL VETERINARY \&

AGRIC UNIV

40 THORVALDSENSVEJ

FREDERIKSBERG C DK-1871

DENMARK

353-528-3354 


\section{D5 REGL SRANTS 1/12/96 OR L__ FER}

HENK SCHOLS

FOOD CHEMISTRY

AGRICULTURAL UNIVERSITY

BOMENWEG 2

WAGENINGEN $670 \mathrm{HD}$

NETHERLANDS

313-748-2239

CAROLYN J. SCHULTZ

SCHOOL OF BOTANY

UNIV OF MELBOURNE

PARKVILLE VICTORIA 3052

AUSTRALIA

039-344-4870

VINCENT J.H. SEWALT

PLANT BIOLOGY DIVISION

SAMUEL ROBERTS NOBLE

FNDN

BOX 2180/2510 SAM NOBLE

PKWY

ARDMORE OK 73402

405-223-5810

\section{ALLAN M. SHOWALTER \\ PLANT BIOLOGY \\ OHIO UNIVERSITY \\ PORTER HALL \\ ATHENS OH 457012979 \\ 614-593-1135}

THOMAS L. SIMS

PLANT MOLECULAR BIOL

CTR

NORTHERN ILLINOIS

UNIVERSITY

325 MONTGOMERY HALL

DE KALB IL 60115

815-753-7817

KRISTEN L SMITH

CHEMISTRY

OHIO UNIVERSITY

424 CARRIAGE HILL DRIVE

ATHENS OH 45701

614-593-9466

SIMON G. SOUTHERTON

ANC 092424085

FORBIO RESEARCH PTY LTD

50 MEIERS ROAD

INDOOROOPILLY

QUEENSLAND 4068

AUSTRALIA

7-870-5888

CHRIS J STAIGER

BIOLOGICAL SCIENCES

PURDUE UNIVERSITY

1392 LILLY HALL

WEST LAFAYETTE IN 47907

317-496-1769
STEVEN R. THOMAS

APPLIED BIOL SCIENCE

NATIONAL RENEWABLE

ENERGY LAB

1617 COLE BOULEVARD

GOLDEN CO 80401

303-275-3858

MARY L. TIERNEY

BOTANY

UNIV OF VERMONT

120 MARSH LIFE SCIENCE

BLDG

BURLINGTON VT 05405

802-656-0434

DERI TOMOS

BIOLOGICAL SCIENCES

UNIV OF WALES

BANGOR GWYNEDD WALES

LL57. 2UW

UK

124-838-2362

YUZURU TOZAWA

DEPT OF CROP BREEDING

NATIONAL AGRICULTURE

RSCH CTR

3-1-1 KANNONDAI, TSUKUBA

IBARAKI 305

JAPAN

29-838-8949

EDITH P.W.M. VAN DOOREN

PLANT BIOTECHNOLOGY TNO

CENTER FOR PHYTOTECHNO

RUL-TNO

WASSENAARSEWEG 64

LEIDEN 2333 AL

NETHERLANDS

71-527-4932

CLAUDIA E. VERGARA

BOTANY/PLANT PATHOLOGY

PURDUE UNIVERSITY

WEST LAFAYETTE

WEST LAFAYETTE IN 47907

317-494-6140

JEAN-PAUL VINCKEN

FOOD SCIENCE

WAGENINGEN

AGRICULTURAL UNIV

PO BOX 8129

WAGENINGEN $6700 \mathrm{EV}$

NETHERLANDS

83-708-4897
JIM L WADE

CORPORATE RESEARCH

HERCULES INC

500 HERCULES ROAD

WILMINGTON DE 19808

302-995-4216

MICHAEL H. WALTER

INST OF PLANT PHYSIOL 260

UNIV OF HOHENHEIM

BOX 7005 62/EMIL WOLFFSTR

25

STUTTGART 70 D-70593

GERMANY

711-459-2199

ROLAND M. WELLE

PIONEER HI-BRED

APENSENERSTR 198

BUXTEHUDE D-21614

GERMANY

4i6-173-7363

ZEV M. WINICUR

MCD BIOLOGY

UNIV OF COLORADO

CAMPUS BOX 347

BOULDER CO 80309

303-492-8893

HO-HYUNG WOO

PLANT.PATHOLOGY

UNIV OF ARIZONA

FORBES BLDG 204

TUCSON AZ 85721

602-621-3917

BRANDON A. WUSTMAN

BIOLOGICAL SCIENCES

MICHIGAN TECHNOLOGICAL

UNIV

HOUGHTON MI 49931

906-487-3223

OLGA ZABOTINA

LAB OF CELL WALL BIOCHEM INST OF BIOLOGY

PO BOX 299

KAZAU 420503

RUSSIA

843-238-7348

IGNACIO ZARRA

BIOLOGIA VEGETAL

UNIVERSIDAD DE SANTIAGO

FAC DE BIOLOGIA

SANTIAGO DE COMPOSTELA

15706

SPAIN

348-156-3100 